\title{
Transcriptional profiling analysis predicts potential biomarkers for glaucoma: HGF, AKR1B10 and AKR1C3
}

\author{
QIAOLI NIE $^{1}$ and XIAOYAN ZHANG ${ }^{2}$ \\ ${ }^{1}$ Department of Ophthalmology, The Second People's Hospital of Jinan, Jinan, Shandong 250001; \\ ${ }^{2}$ Department of Ophthalmology, The People's Hospital of Shouguang, Weifang, Shandong 262700, P.R. China
}

Received October 13, 2017; Accepted July 26, 2018

DOI: 10.3892/etm.2018.6875

\begin{abstract}
Glaucoma results in damage to the optic nerve and vision loss. The aim of this study was to screen more accurate biomarkers and targets for glaucoma. The datasets E-GEOD-7144 and E-MEXP-3427 were screened for differently expressed genes (DEGs) by significance analysis of microarrays. Functional and pathway enrichment analysis were processed. Pathway relationship networks and gene co-expression networks were constructed. DEGs of disease and treatment with the same symbols were of interest. RT-qPCR was processed to verify the expression of key DEGs. A total of 1,019 DEGs of glaucoma were identified and 93 DEGs in transforming growth factor- $\beta 1$ (TGF- $\beta 1$ ) and TGF- $\beta 1-2$ treatment cases compared with the normal control group. Pathway relationship network of glaucoma was constructed with 25 nodes. The pathway relationship network of TGF- $\beta 1$ and -2 treatment groups was constructed with 11 nodes. Glaucoma-related DEGs in GO terms and pathways were inserted and 180 common DEGs were obtained. Then, gene co-expression network of glaucoma-related DEGs was constructed with 91 nodes. Furthermore, DEGs of TGF- $\beta 1$ and -2 treated glaucoma in GO terms and pathways were inserted, and 29 common DEGs were identified. Based on these DEGs, gene co-expression network was constructed with 12 nodes and 16 edges. Finally, a total of 6 important DEGs of disease and treatment were inserted and obtained. They were HGF, AKR1B10, AKR1C3, PPAP2B, INHBA and BCAT1. The expression of HGF, AKR1B10 and AKR1C3 was decreased in glaucoma samples and treatment samples. In conclusion, HGF, AKR1B10 and AKR1C3 may be key genes for glaucoma diagnosis and treatment.
\end{abstract}

\section{Introduction}

Glaucoma includes various types of open-angle, closed-angle and normal-tension glaucoma, and results in damage to the

Correspondence to: Dr Qiaoli Nie, Department of Ophthalmology, The Second People's Hospital of Jinan, 148 Jingyi Road, Huaiyin, Jinan, Shandong 250001, P.R. China

E-mail: nieqiaoli123@126.com

Key words: glaucoma, biomarker, TGF- $\beta 1$ and -2, pathway optic nerve and vision loss (1). The risk factors of glaucoma include increased pressure in the eye and family history of high blood pressure, obesity and migraines (2). Once vision loss from glaucoma occurs, it will be permanent (3). Therefore, the exact pathogenesis requires research for prevention, diagnosis and treatment of glaucoma.

Several important genes and their molecular mechanisms have been found to be related with glaucoma. The research of Skarie and Link revealed that WDR36 has a negative correlation with $\mathrm{p} 53$ stress-response pathway in the primary open-angle glaucoma (4). In a large animal model, MMP-1 was confirmed to lower the intraocular pressure of glaucoma cases induced by steroid (5). Besides, LOXL1 indicates high prevalence of glaucoma, but with low specificity (6). Jelodari-Mamaghani et al (7) suggested that some sequence variations of latent transforming growth factor- $\beta$ (TGF- $\beta$ ) binding protein 2 could induce primary open-angle glaucoma and other pseudoexfoliation syndromes. However, some genes are controversial because their underlying molecular mechanisms were uncovered. Kumar et al (8) showed that $3.59 \%$ of Indian primary open-angle glaucoma patients had mutations in MYOC, OPIT and CY1B1 genes. But in some primary congenital glaucoma patients, MYOC and FOXC1 mutations did not participate (9). Though various genes and related molecular mechanisms were investigated, targeted therapy for glaucoma has not achieved a breakthrough. Accurate targets for glaucoma are urgently needed.

Bioinformatics analysis was processed to screen more accurate biomarkers and therapy targets for glaucoma. Most importantly, two different datasets related to the disease and treatment were downloaded. Various networks including pathway-relationship network and gene co-expression networks were constructed. The final intersection was identified to achieve the goal and lay a theoretical foundation for further research.

\section{Materials and methods}

Microarray datasets. Interesting datasets for glaucoma were searched in ArrayExpress Archive (http://www.ebi.ac.uk/ arrayexpress/). Two different datasets were obtained, including E-GEOD-7144 and E-MEXP-3427 (10). The platforms of the databases were [HG-U133A] Affymetrix Human Genome U133A Array and Affymetrix GeneChip Human Genome 
U133 Plus 2.0 [HG-U133_Plus_2], respectively. The expression profiling of E-GEOD-7144 contains 6 trabecular meshwork samples of glaucoma patients, including 2 glaucoma samples treated with ethyl alcohol as the normal control group and 4 glaucoma samples treated by TGF- $\beta 1$ and -2 . The expression profile of E-MEXP-3427 contained 9 trabecular meshwork samples, including 2 glaucoma samples and 7 controls.

Data pretreatment and identification of DEGs. The downloaded raw data were corrected and normalized by Robust Multi-chip Average (RMA) algorithm (11), and then annotated based on Affymetrix (http://www.affymetrix.com/support/ technical/annotationfilesmain.affx). Following, normalized unscaled standard errors (NUSE) controlling was used for quality control (12). Compared with the normal control group, the DEGs in glaucoma samples were screened by significance analysis of microarrays (SAM) (13). Similarly, the DEGs in TGF- $\beta 1$ and -2 treatment groups compared with the untreated control group were also identified by this method. The threshold of DEGs in the two groups was $\mathrm{P}<0.05$ and $\log \mathrm{FCl}>2$.

Gene Ontology (GO) functional enrichment and Kyoto Encyclopedia of Genes and Genomes (KEGG) pathway enrichment analysis. GO database was built by Gene Ontology Consortium, which described gene and their protein functions (14). Based on this database, screened DEGs in the two groups were functional annotated, and then significant GO terms were selected by Fisher exact test and multiple hypothesis test with the threshold of false discovery rate (FDR) $<0.05$. Beniamini-Hochberg (BH) method was utilized for correcting P-value to FDR (15).

Based on KEGG database, the screened DEGs in the two groups were gathered in eight categories, including total network, metabolic process, genetic information transfer, environmental information transfer, intracellular biological process, biological systems, human disease and drug development (16). The enriched pathways were calculated by Fisher's exact test with the criteria of FDR $<0.05$.

Construction of pathway relationship network. KEGG database provides the relationship of genes and pathways. In this study, the pathway relationship network was constructed based on this database. According to the information of signal transduction in the network, upstream and downstream signal pathways were obtained.

Intersection of DEGs in GO terms and pathways. DEGs in GO terms and pathways with the same symbols were of interest, and common DEGs of the two groups were obtained.

Gene co-expression network construction. Gene co-expression network reflected the regulatory relationship among DEGs, and showed the hub nodes with higher degrees. This network was constructed with the threshold of correlation coefficient $>1$.

Intersection of DEGs of disease and treatment. DEGs of disease and treatment with the same symbols were of interest, and the important DEGs were obtained.
Trabecular meshwork cell culture and treatment. As described in previous studies, primary culture of HTM-2 cells was prepared from glaucoma and normal donors. The experiment included 3 groups: the normal control group, the disease group and the treatment group. All cells were maintained at $37^{\circ} \mathrm{C}$ in $5 \% \mathrm{CO}_{2}$ in low glucose Dulbecco's modified Eagle's medium (DMEM) with $10 \%$ fetal bovine serum. All reagents were purchased from Invitrogen; Thermo Fisher Scientific, Inc. (Waltham, MA, USA). After $24 \mathrm{~h}$ treatment, HTM cells from glaucoma in the treatment group were subcultured in free culture medium for $48 \mathrm{~h}$, and then treated by TGF- $\beta 1$ and $-2(1 \mathrm{ng} / \mathrm{ml}$ of free culture medium) for $1 \mathrm{~h}$. The cells in the normal control and disease groups were also processed by the above steps but treated by normal saline. The experiment design with three replications was used.

Determination of expression levels of important DEGs by $R T-q P C R$. Total RNA of cells in the three groups were reverse transcribed by oligo(dT) primer and Superscript II Reverse Transcriptase (Invitrogen; Thermo Fisher Scientific, Inc.). The primers of DEGs were designed as follows: HGF (upstream) 5'-ACAGCTTTTTGCCTTCGAGCTATCGGGGTAAAGAC CTACAGG-5'; (downstream) 5'-CATCAAAGCCCTTATCG GGGATA-3'; AKR1B10 (upstream) 5'-GGACCTGTTCAT CGTCAGCAA-3'; (downstream) 5'-CCCCAGACTTGAATC CCTGTG-3'; AKR1B10 (upstream) 5'-GTAAAGCTTTGGAG GTCAC-3'; (downstream) 5'-CACCCATCGTTTGTCTCGT-3'; PPAP2B2B (upstream) 5'-CCTCTTCTGCCTCTTCATGG-3'; (downstream) 5'-GCCACATACGGGTTCTGAGT-3'; GAPDH (upstream) 5'-AATGCATCCTGCACCACCAA-3'; (downstream) 5'-GTAGCCATATTCATTGTCATA-3'.

All amplifications were performed on the Rotor-Gene 3000 real-time cycler (Corbett Research, Sydney, Australia) instrument. The reaction conditions were 1 cycle of $95^{\circ} \mathrm{C}$ for $10 \mathrm{~min}, 42$ cycles of $95^{\circ} \mathrm{C}$ for $20 \mathrm{sec}$, and $60^{\circ} \mathrm{C}$ for $20 \mathrm{sec}$ and $72^{\circ} \mathrm{C}$ for $20 \mathrm{sec}$. SYBR Premix Ex Taq ${ }^{\mathrm{TM}}$ (Takara, Otsu, Japan) was used in the following PCR procedure. The experiment was repeated three times.

This study has been approved by the Ethics Committee of The Second People's Hospital of Jinan (Jinan, China) and all patients provided informed consent.

\section{Results}

Identification of DEGs. Compared with the normal control group, a total of 1019 DEGs of glaucoma were identified, including 471 up- and 548 downregulated genes. Similarly, total 93 DEGs in TGF- $\beta 1$ and 2 treatment cases compared with the untreated control group were obtained, including 71 upregulated and 22 downregulated DEGs.

Functional and pathway enrichment analysis. As shown in Table I, the screened glaucoma-related DEGs were enriched in various GO terms, including negative regulation of cell proliferation $(\mathrm{FDR}=9.56 \mathrm{E}-11)$, endoplasmic reticulum unfolded protein response $(\mathrm{FDR}=9.56 \mathrm{E}-11)$, activation of signaling protein activity involved in unfolded protein response (FDR=7.04E-08) and cellular protein metabolic process (FDR=4.22E-07). Simultaneously, these DEGs involved in pathways, such as protein processing in endoplasmic 
Table I. The top 5 GO and KEGG pathways enriched by glaucoma-related DEGs.

\begin{tabular}{|c|c|c|c|c|c|}
\hline GO ID & GO name & $\begin{array}{l}\text { Diff gene } \\
\text { counts in GO }\end{array}$ & $\begin{array}{l}\text { Enrichment } \\
\text { score }\end{array}$ & P-value & FDR \\
\hline GO:0008285 & Negative regulation of cell proliferation & 32 & 5.306158859 & $5.10 \times 10^{-14}$ & $9.56 \times 10^{-11}$ \\
\hline GO:0030968 & $\begin{array}{l}\text { Endoplasmic reticulum unfolded } \\
\text { protein response }\end{array}$ & 17 & 12.15861552 & $8.56 \times 10^{-14}$ & $9.56 \times 10^{-11}$ \\
\hline GO:0006987 & $\begin{array}{l}\text { Activation of signaling protein activity } \\
\text { involved in unfolded protein response }\end{array}$ & 13 & 12.05803873 & $9.46 \times 10^{-11}$ & $7.04 \times 10^{-8}$ \\
\hline GO:0044267 & Cellular protein metabolic process & 32 & 3.688553148 & $7.55 \times 10^{-10}$ & $4.22 \times 10^{-7}$ \\
\hline GO:0007050 & Cell cycle arrest & 15 & 6.956560809 & $9.88 \times 10^{-9}$ & $4.22 \times 10^{-6}$ \\
\hline Pathway ID & Pathway name & $\begin{array}{l}\text { Diff gene counts } \\
\text { in pathway }\end{array}$ & $\begin{array}{l}\text { Enrichment } \\
\text { score }\end{array}$ & P-value & FDR \\
\hline 4141 & $\begin{array}{l}\text { Protein processing in endoplasmic } \\
\text { reticulum }\end{array}$ & 21 & 7.464764652 & $2.17 \times 10^{-12}$ & $4.74 \times 10^{-10}$ \\
\hline 4151 & PI3K-Akt signaling pathway & 25 & 4.276848144 & $3.28 \times 10^{-9}$ & $3.51 \times 10^{-7}$ \\
\hline 5200 & Pathways in cancer & 24 & 4.356891907 & $4.81 \times 10^{-9}$ & $3.51 \times 10^{-7}$ \\
\hline 1100 & Metabolic pathways & 45 & 2.246694155 & $1.70 \times 10^{-6}$ & $9.32 \times 10^{-5}$ \\
\hline 5410 & Hypertrophic cardiomyopathy (HCM) & 9 & 6.285457295 & $2.74 \times 10^{-5}$ & $1.06 \times 10^{-3}$ \\
\hline
\end{tabular}

DEGs, differently expressed genes; GO, Gene Ontology; KEGG, Kyoto Encyclopedia of Genes and Genomes; FDR, false discovery rate.

Table II. The top 5 GO and KEGG pathways enriched by TGF- $\beta 1$ and -2 treatment-related DEGs.

\begin{tabular}{|c|c|c|c|c|c|}
\hline GO ID & GO name & $\begin{array}{c}\text { Diff gene } \\
\text { counts in GO }\end{array}$ & $\begin{array}{l}\text { Enrichment } \\
\text { score }\end{array}$ & P-value & FDR \\
\hline GO:0044597 & Daunorubicin metabolic process & 4 & 327.380597 & $6.92 \times 10^{-10}$ & $1.77 \times 10^{-7}$ \\
\hline GO:0044598 & Doxorubicin metabolic process & 4 & 327.380597 & $6.92 \times 10^{-10}$ & $1.77 \times 10^{-7}$ \\
\hline GO:0071395 & Cellular response to jasmonic acid stimulus & 3 & 491.0708955 & $2.72 \times 10^{-8}$ & $4.63 \times 10^{-6}$ \\
\hline GO:0030198 & Extracellular matrix organization & 7 & 21.82537313 & $7.10 \times 10^{-8}$ & $9.06 \times 10^{-6}$ \\
\hline GO:0051897 & $\begin{array}{l}\text { Positive regulation of protein } \\
\text { kinase B signaling cascade }\end{array}$ & 5 & 48.14420544 & $1.38 \times 10^{-7}$ & $1.41 \times 10^{-5}$ \\
\hline Pathway ID & Pathway name & $\begin{array}{l}\text { Diff gene counts } \\
\text { in pathway }\end{array}$ & $\begin{array}{l}\text { Enrichment } \\
\text { score }\end{array}$ & $\mathrm{P}$-value & FDR \\
\hline 1100 & Metabolic pathways & 12 & 6.608186988 & $4.52 \times 10^{-7}$ & $2.35 \times 10^{-5}$ \\
\hline 4512 & ECM-receptor interaction & 5 & 37.62995368 & $4.79 \times 10^{-7}$ & $2.35 \times 10^{-5}$ \\
\hline 5146 & Amoebiasis & 5 & 30.03491716 & $1.47 \times 10^{-6}$ & $3.84 \times 10^{-5}$ \\
\hline 4510 & Focal adhesion & 6 & 19.07071439 & $1.57 \times 10^{-6}$ & $3.84 \times 10^{-5}$ \\
\hline 4974 & Protein digestion and absorption & 4 & 29.76187246 & $2.10 \times 10^{-5}$ & $4.12 \times 10^{-4}$ \\
\hline
\end{tabular}

DEGs, differently expressed genes; GO, Gene Ontology; KEGG, Kyoto Encyclopedia of Genes and Genomes; FDR, false discovery rate.

reticulum $(\mathrm{FDR}=4.74 \mathrm{E}-10)$, PI3K-Akt signaling pathway $(\mathrm{FDR}=3.51 \mathrm{E}-07)$, pathways in cancer $(\mathrm{FDR}=3.51 \mathrm{E}-07)$ and metabolic pathways (FDR=9.32E-05).

Similarly, the DEGs of TGF- $\beta 1$ and -2 treatment patients participated in different GO terms, such as daunorubicin metabolic process (FDR=1.76E-07), doxorubicin metabolic process $(\mathrm{FDR}=1.76 \mathrm{E}-07)$, cellular response to jasmonic acid stimulus (FDR=4.62E-06). These DEGs were also enriched in various pathways including metabolic pathways $(\mathrm{FDR}=2.34 \mathrm{E}-05), \mathrm{ECM}-$ receptor interaction $(\mathrm{FDR}=2.34 \mathrm{E}-05)$ and amoebiasis (FDR=3.84E-05) (Table II).

Construction of pathway relationship network. Pathway relationship network of glaucoma was constructed with 25 nodes and 87 edges (Fig. 1). The hub nodes were MAPK signaling pathway (degree 18), apoptosis (degree 18) and cell 


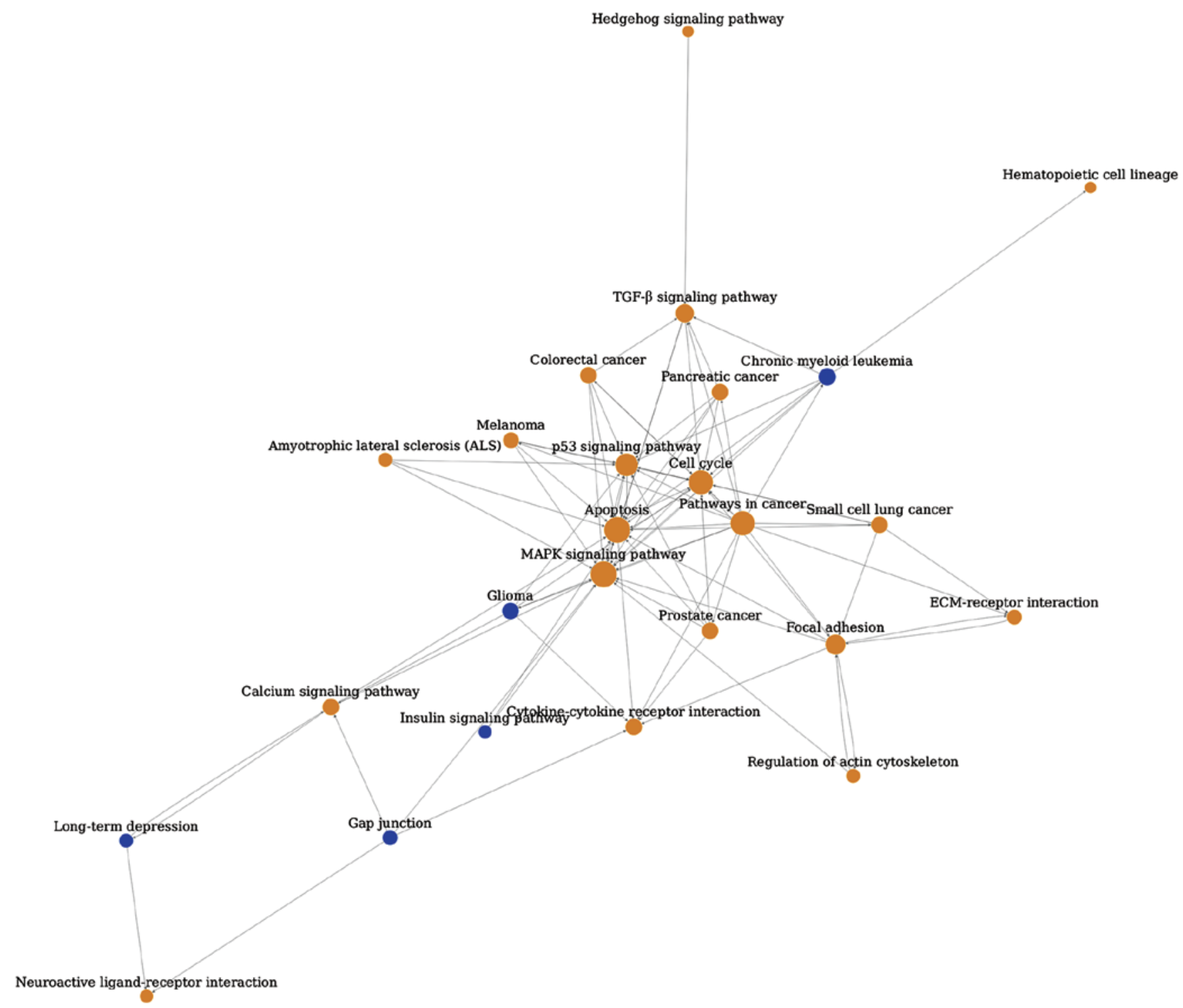

Figure 1. Pathway relationship network of glaucoma-related DEGs. The blue nodes represent pathways involving downregulated DEGs, and the yellow nodes represent pathways involving both up- and downregulated DEGs. The arrows represent regulatory direction. DEG, differently expressed gene.

cycle (degree 15). In addition, several upstream pathways were identified, including pathways in cancer and gap junction. Cytokine-cytokine receptor interaction was shown as a downstream pathway.

As shown in Fig. 2, pathway relationship network of TGF- $\beta 1$ and -2 treatment groups was constructed with 11 nodes and 20 edges. Focal adhesion, pathways in cancer and Wnt signaling pathway were the hub nodes with degree of 7.7 and 6 , respectively.

Gene co-expression network construction of common DEGs. Based on gene symbol, glaucoma-related DEGs in GO terms and pathways were inserted and 180 common DEGs were obtained. Then, gene co-expression network of glaucoma-related DEGs was constructed, including 91 nodes and 166 edges (Fig. 3). In addition, the hub nodes in this network were protein disulfide isomerase family A, member 4 (PDIA4, degree 13), osteosarcoma amplified 9, endoplasmic reticulum lectin (OS9, degree 12), derlin 3 (DERL3, degree 10) and sel-1 suppressor of lin-12-like (SEL1L, degree 9).
Furthermore, DEGs of TGF- $\beta 1$ and -2 treated glaucoma in GO terms and pathways were inserted, and 29 common DEGs were identified. Based on the DEGs, gene co-expression network was constructed with 12 nodes and 16 edges (Fig. 4). The hub node of this network was HGF (degree 4).

Intersection of DEGs of disease and treatment. Finally, a total of 6 important DEGs of disease and treatment were inserted and obtained. They were hepatocyte growth factor (HGF), aldo-ketoreductase family 1, member B10 (AKR1B10), aldo-ketoreductase family 1, member C3 (AKR1C3), phosphatidic acid phosphatase type $2 \mathrm{~B}$ (PPAP2B), inhibin $\beta \mathrm{A}$ (INHBA) and branched chain amino-acid transaminase 1, cytosolic (BCAT1). These genes may be biomarkers and targets for glaucoma diagnosis and treatment.

$D E G$ expression level by $R T-q P C R$. The expression level of HGF, AKR1B10, AKR1C3 and PPAP2B were determined by RT-qPCR. As shown in Fig. 5, the expression of HGF, AKR1B10 and AKR1C3 was significantly decreased in 


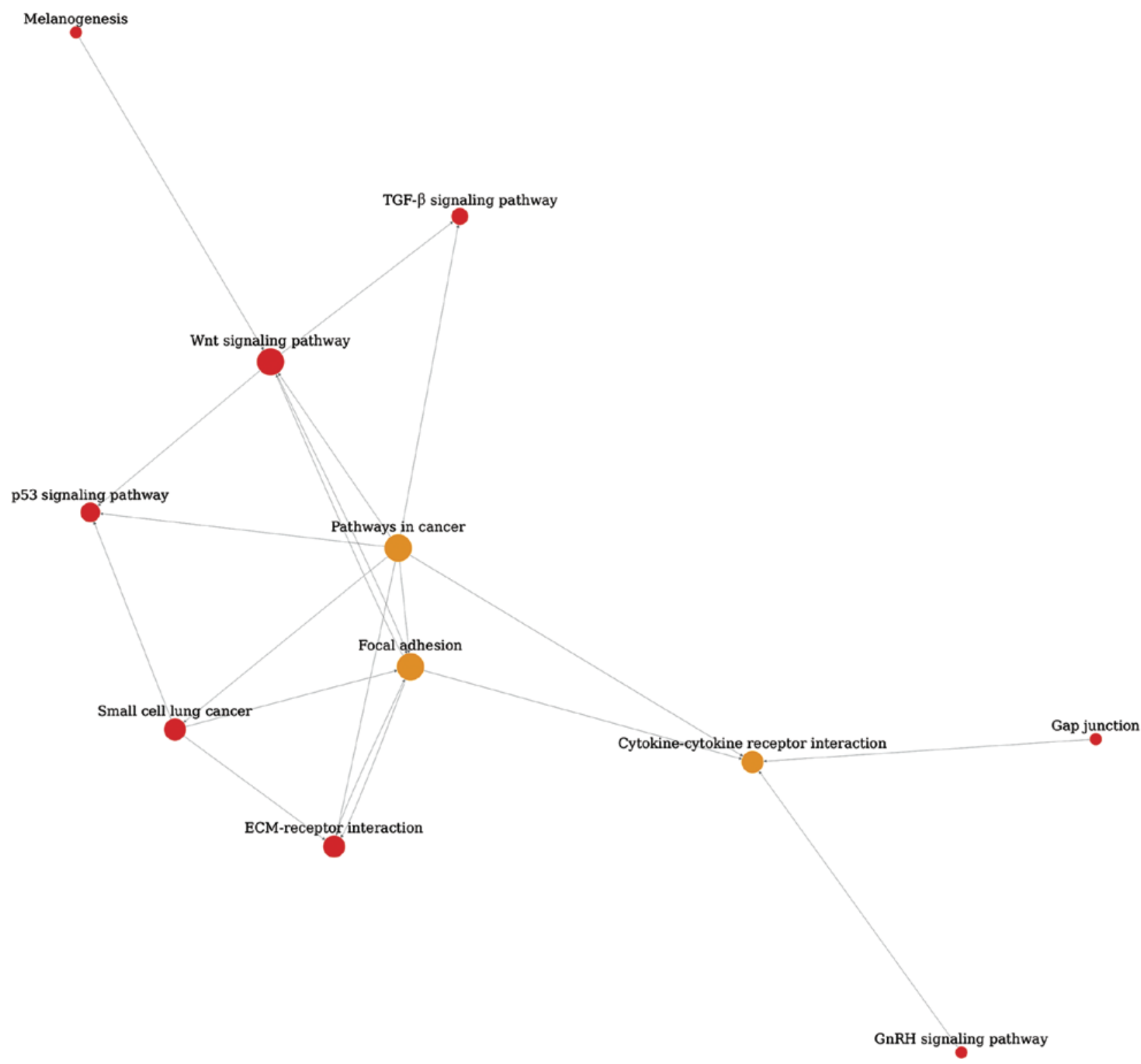

Figure 2. Pathway relationship network of TGF- $\beta 1$ and -2 treatment-related DEGs. The red nodes represent pathways involving upregulated DEGs, and the yellow nodes represent pathways involving both up- and downregulated DEGs. The arrows represent regulatory direction. DEG, differently expressed gene; TGF- $\beta 1$, transforming growth factor- $\beta 1$.

glaucoma and treatment samples. However, no significant difference of AKR1C3 was found in the three groups.

\section{Discussion}

Glaucoma is treated by daily eye drop drugs, but with unsatisfactory results (17). Gene therapy has successfully progressed for other eye diseases, and may be an effective method for the treatment of glaucoma in the future (17). In this study, various DEGs were screened and their association with glaucoma was examined. We also examined DEGs related to treatment, including HGF, AKR1B10, AKR1C3 and PPAP2B.

HGF encodes a protein which activates a tyrosine kinase signaling cascade and further regulated cell growth, motility and morphogenesis (18). In the patients with eyes suffering from primary open-angle glaucoma, the concentration of HGF was significantly elevated (19). In addition, in 2010, four SNPs were found closely related with primary angle closure glaucoma, including rs12536657, rs17427817, rs5745718 and rs12540393 (20). Moreover, the level of HGF was confirmed to stimulate the expression of MMP-1, and further affected the migration of human corneal epithelial cells (21). In this study, the gene was involved in various functions and pathways, including the negative regulation of the apoptotic process, mitosis, PI3K-Akt signaling pathway and pathways in cancer. In addition, the expression of HGF was significantly lower in the glaucoma and treatment groups than in the normal control group. In a previous study, the aqueous humor factors significantly affected mitosis in the molecular pathogenesis of primary open-angle glaucoma (22). In various diseases, HGF has been confirmed to be an effective target of TGF- $\beta 1$ and -2 treatments. For example, tumor-derived TGF- $\beta 1$ promoted HGF-dependent invasion of squamous carcinoma cells (23). Furthermore, TGF- $\beta$ was involved in HGF-c-Met pathway, and then induced oligodendrocyte precursor cell chemotaxis (24). Besides, Tripathi et al (25) found that the level of TGF- $\beta 2$ was 


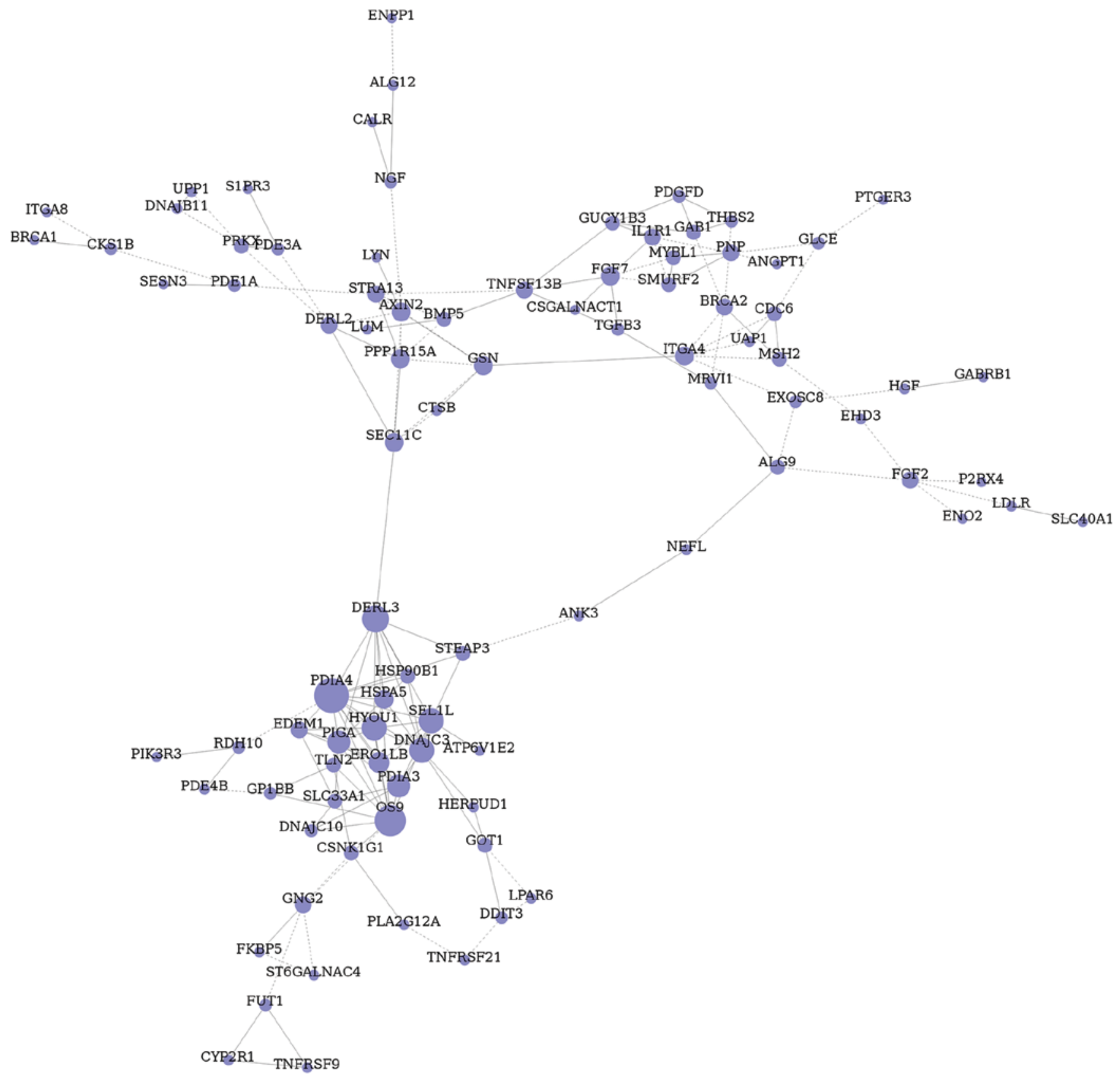

Figure 3. Gene co-expression network of important glaucoma-related DEGs. The solid lines represent DEGs with positive correlation, and the dotted lines represent DEGs with negative correlation. DEG, differently expressed gene.

significantly increased in aqueous humor in glaucomatous eyes. Overall, HGF is an important biomarker for glaucoma and also a key target for glaucoma treatment.

Another key DEG, AKR1B10, was also identified in both glaucoma and TGF- $\beta 1$ and -2 treatment-related gene co-expression network. This gene encoded a member of aldo/ketoreductase superfamily, which could effectively reduce aliphatic and aromatic aldehydes. As known, NADPH-dependent aldo-ketoreductase is an important rate limiting enzyme of polyol pathway, which accelerates glucose metabolism and also affects the diabetic cataract and retinopathy (26). In this study, AKR1B10 was found enriched in the daunorubicin metabolic process, doxorubicin metabolic process, and farnesol catabolic process. As shown in previous results, intraoperative daunorubicin could decrease the intraocular pressure safely and effectively in high-risk surgical cases of glaucoma (27). Moreover, doxorubicin was also confirmed to be an effective adjunct to glaucoma surgery (28). Thereby, we inferred that AKR1B10 participated in the pathogenesis of glaucoma by being involved in the daunorubicin and doxorubicin metabolic process. Interestingly, AKR1C3 and AKR1B10 had positive relationships in the gene co-expression network of glaucoma. AKR1C3 is also a member of the aldo-ketoreductase family 1. Besides the daunorubicin and doxorubicin metabolic process, AKR1C3 is enriched in immune response, G-protein coupled receptor signaling pathway and positive regulation of protein kinase B signaling cascade. Among these functions 


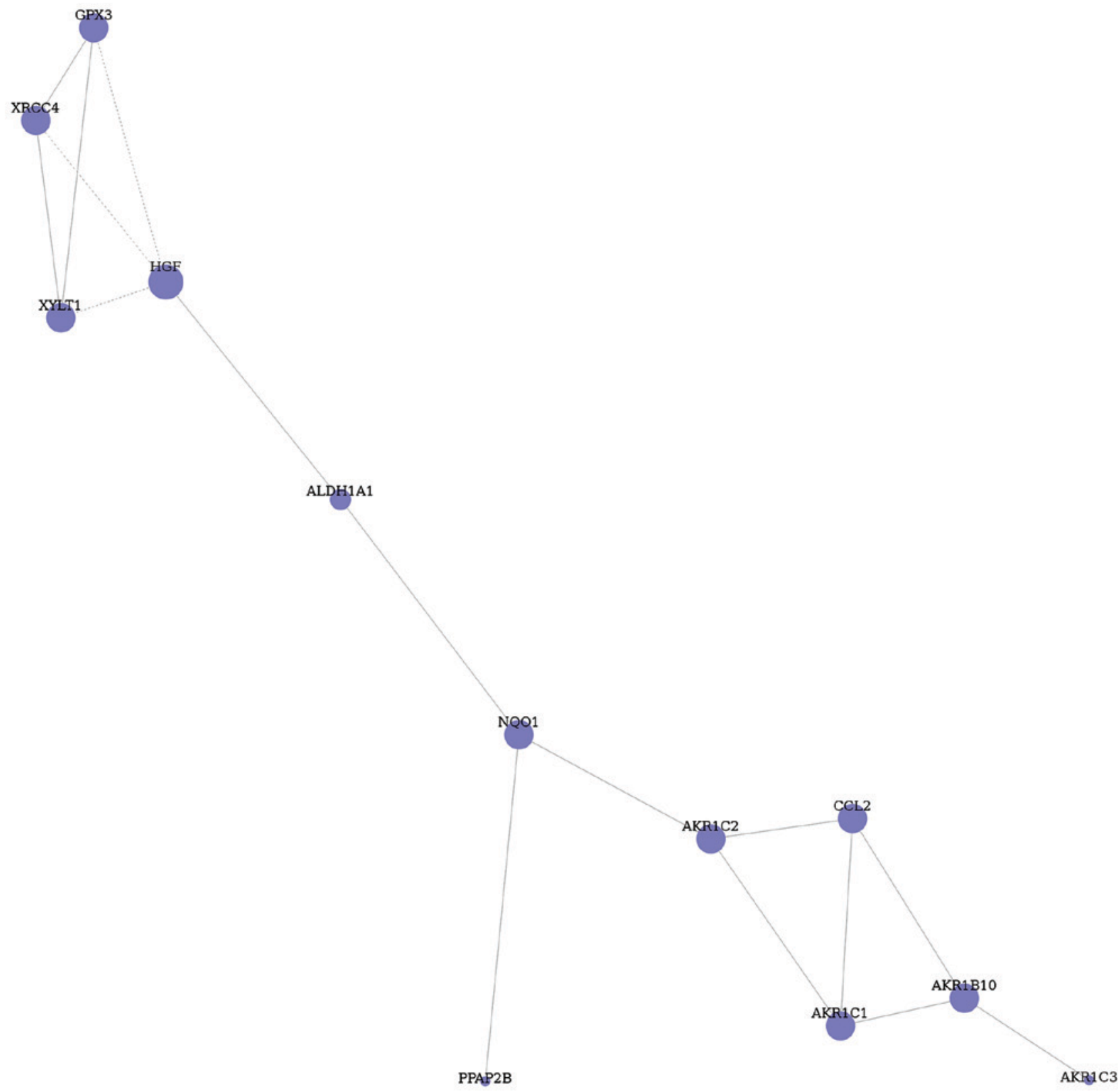

Figure 4. Important gene co-expression network TGF- $\beta 1$ and -2 treatment-related DEGs. The solid lines represent DEGs with positive correlation, and the dotted lines represent DEGs with negative correlation. DEG, differently expressed gene; TGF- $\beta 1$, transforming growth factor- $\beta 1$.

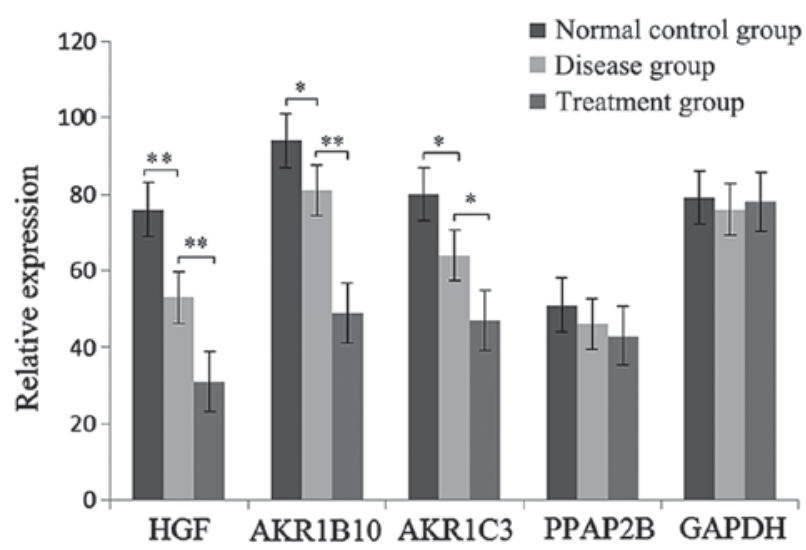

Figure 5. The expression level of HGF, AKR1B10, AKR1C3 and PPAP2B. HGF, hepatocyte growth factor; AKR1B10, aldo-ketoreductase family 1 , member B10; AKR1C3, aldo-ketoreductase family 1, member C3; PPAP2B, phosphatidic acid phosphatase type $2 \mathrm{~B} .{ }^{*} \mathrm{p}<0.05,{ }^{* *} \mathrm{p}<0.01$. and pathways, immune response, especially oxidative stress, played a key role in keeping a physiological balance by participating in the pathogenic cellular processes of glaucoma (29). In addition, G-protein activation mediated by shear stress was confirmed to be a possible step in molecular mechanism of glaucoma formation (30). Most importantly, the expression of AKR1B10 and AKR1C3 was significantly lower in the glaucoma and treatment groups than in the normal control group. According to the above information, AKR1C3 was inferred to be a key gene for prevention and treatment of glaucoma.

Simultaneously, PPAP2B was screened with a higher degree in this study and also involved in different functions and pathways, including small molecule metabolic process, negative regulation of protein phosphorylation and $F c \gamma R$ mediated phagocytosis. PPAP2B encoded a member of the 
phosphatidic acid phosphatase family which participate in the formation of diacylglycerol and glycerolipids (31). Moreover, Chiasseu et al (32) showed that the signature pathological features of glaucoma included altered phosphorylation of tauopathies. Besides, various metabolic abnormalities such as carbohydrate and uric acid metabolic abnormalities were confirmed to play an important role in pathogenesis of glaucoma damage (33). Thereby, PPAP2B may be a key gene in the pathogenesis of glaucoma through its involvement in the small molecule metabolic process. However, no significant difference of PPAP2B was found in the three groups.

In conclusion, HGF, AKR1B10 and AKR1C3 may be key genes for glaucoma diagnosis and treatment.

\section{Acknowledgements}

Not applicable.

\section{Funding}

No funding was received.

\section{Availability of data and materials}

The datasets used and/or analyzed during the current study are available from the corresponding author on reasonable request.

\section{Authors' contributions}

QN constructed pathway relationship network. XZ helped with gene co-expression network construction. QN and XZ were also involved in the conception and design of the study. Both authors have read and approved the final manuscript.

\section{Ethics approval and consent to participate}

This study has been approved by the Ethics Committee of The Second People's Hospital of Jinan (Jinan, China). All patients in this study provided informed consent.

\section{Patient consent for publication}

Not applicable.

\section{Competing interests}

The authors declare that they have no competing interests.

\section{References}

1. Martus P, Stroux A, Budde WM, Mardin CY, Korth M and Jonas JB: Predictive factors for progressive optic nerve damage in various types of chronic open-angle glaucoma. Am J Ophthalmol 139: 999-1009, 2005.

2. Graham SL, Butlin M, Lee M and Avolio AP: Central blood pressure, arterial waveform analysis, and vascular risk factors in glaucoma. J Glaucoma 22: 98-103, 2013.

3. Curriero FC, Pinchoff J, van Landingham SW, Ferrucci L, Friedman DS and Ramulu PY: Alteration of travel patterns with vision loss from glaucoma and macular degeneration. JAMA Ophthalmol 131: 1420-1426, 2013.
4. Skarie JM and Link BA: The primary open-angle glaucoma gene WDR36 functions in ribosomal RNA processing and interacts with the p53 stress-response pathway. Hum Mol Genet 17: 2474-2485, 2008

5. Borrás T, Buie LK and Spiga MG: Inducible scAAV2.GRE. MMP1 lowers IOP long-term in a large animal model for steroidinduced glaucoma gene therapy. Gene Ther 23: 438-449, 2016.

6. Fan BJ and Wiggs JL: Glaucoma: Genes, phenotypes, and new directions for therapy. J Clin Invest 120: 3064-3072, 2010.

7. Jelodari-Mamaghani S, Haji-Seyed-Javadi R, Suri F, Nilforushan N, Yazdani S, Kamyab K and Elahi E: Contribution of the latent transforming growth factor- $\beta$ binding protein 2 gene to etiology of primary open angle glaucoma and pseudoexfoliation syndrome. Mol Vis 19: 333-347, 2013.

8. Kumar A, Basavaraj MG, Gupta SK, Qamar I, Ali AM, Bajaj V, Ramesh TK, Prakash DR, Shetty JS and Dorairaj SK: Role of CYP1B1, MYOC, OPTN, and OPTC genes in adult-onset primary open-angle glaucoma: Predominance of CYP1B1 mutations in Indian patients. Mol Vis 13: 667-676, 2007.

9. Tanwar M, Kumar M, Dada T, Sihota R and Dada R: MYOC and FOXC1 gene analysis in primary congenital glaucoma. Mol Vis 16: 1996-2006, 2010.

10. Kennedy KD, AnithaChristy SA, Buie LK and Borrás T: Cystatin a, a potential common link for mutant myocilin causative glaucoma. PLoS One 7: e36301, 2012.

11. Keller PJ, Arendt LM, Skibinski A, Logvinenko T, Klebba I, Dong S, Smith AE, Prat A, Perou CM, Gilmore H, et al: Defining the cellular precursors to human breast cancer. Proc Natl Acad Sci USA 109: 2772-2777, 2012.

12. Zheng-Bradley X, Rung J, Parkinson $H$ and Brazma A: Large scale comparison of global gene expression patterns in human and mouse. Genome Biol 11: R124, 2010.

13. Grace $C$ and Nacheva EP: Significance analysis of microarrays (SAM) offers clues to differences between the genomes of adult Philadelphia positive ALL and the lymphoid blast transformation of CML. Cancer Inform 11: 173-183, 2012.

14. Harris MA, Clark J, Ireland A, Lomax J, Ashburner M, Foulger R, Eilbeck K, Lewis S, Marshall B, Mungall C, et al: The Gene Ontology (GO) database and informatics resource. Nucleic Acids Res 32: D258-D261, 2004.

15. Kechris KJ, Biehs B and Kornberg TB: Generalizing moving averages for tiling arrays using combined p-value statistics. Stat Appl Genet Mol Biol 9: Article 29, 2010.

16. Tanabe $M$ and Kanehisa M: Using the KEGG database resource. Curr Protoc Bioinformatics 1: 12, 2012.

17. Quigley HA: Glaucoma. Lancet 377: 1367-1377, 2011.

18. Baykal C, Demirtas E, Al A, Ayhan A, Yuce K, Tulunay G, Kose MF and Ayhan A: Comparison of HGF (hepatocyte growth factor) levels of epithelial ovarian cancer cyst fluids with benign ovarian cysts. Int J Gynecol Cancer 13: 771-775, 2003.

19. Engel LA, Muether PS, Fauser S and Hueber A: The effect of previous surgery and topical eye drops for primary open-angle glaucoma on cytokine expression in aqueous humor. Graefes Arch Clin Exp Ophthalmol 252: 791-799, 2014.

20. Awadalla MS, Thapa SS, Burdon KP, Hewitt AW and Craig JE: The association of hepatocyte growth factor (HGF) gene with primary angle closure glaucoma in the Nepalese population. Mol Vis 17: 2248-2254, 2011.

21. Daniels JT, Limb GA, Saarialho-Kere U, Murphy G and Khaw PT: Human corneal epithelial cells require MMP-1 for HGF-mediated migration on collagen I. Invest Ophthalmol Vis Sci 44: 1048-1055, 2003.

22. Jiang YQ, Nagy RM and Spaeth GL: Effect of aqueous humor factors on the inhibition or enhancement of mitosis: An exploration of pathogenesis of primary open-angle glaucoma. Chin Med J (Engl) 98: 833-834, 1985.

23. Lewis MP, Lygoe KA, Nystrom ML, Anderson WP, Speight PM, Marshall JF and Thomas GJ: Tumour-derived TGF-beta1 modulates myofibroblast differentiation and promotes HGF/SF-dependent invasion of squamous carcinoma cells. Br J Cancer 90: 822-832, 2004.

24. Lalive PH, Paglinawan R, Biollaz G, Kappos EA, Leone DP, Malipiero U, Relvas JB, Moransard M, Suter T and Fontana A: TGF-beta-treated microglia induce oligodendrocyte precursor cell chemotaxis through the HGF-c-Met pathway. Eur J Immunol 35: 727-737, 2005.

25. Tripathi RC, Li J, Chan WF and Tripathi BJ: Aqueous humor in glaucomatous eyes contains an increased level of TGF-beta 2. Exp Eye Res 59: 723-727, 1994. 
26. Huang SP, Palla S, Ruzycki P, Varma RA, Harter T, Reddy GB and Petrash JM: Aldo-keto reductases in the eye. J Ophthalmol 2010: 521204, 2010

27. Varma D, Sihota R and Agarwal HC: Evaluation of efficacy and safety of daunorubicin in glaucoma filtering surgery. Eye (Lond) 21: 784-788, 2007.

28. Ren JM: Experimental study on doxorubicin as an adjunct to glaucoma surgery. Zhonghua Yan Ke Za Zhi 25: 351-354, 1989 (In Chinese)

29. Tezel G: The immune response in glaucoma: A perspective on the roles of oxidative stress. Exp Eye Res 93: 178-186, 2011

30. To CH, Kong CW, Chan CY, Shahidullah M and Do CW: The mechanism of aqueous humour formation. Clin Exp Optom 85: 335-349, 2002.

31. Wu C, Huang RT, Kuo CH, Kumar S, Kim CW, Lin YC, Chen YJ, Birukova A, Birukov KG and Dulin NO, et al: Mechanosensitive PPAP2B regulates endothelial responses to atherorelevant hemodynamic forces. Circ Res 117: e41-e53, 2015.
32. Chiasseu M, Cueva Vargas JL, Destroismaisons L, Vande Velde C, Leclerc N and Di Polo A: Tau accumulation, altered phosphorylation, and missorting promote neurodegeneration in glaucoma. J Neurosci 36: 5785-5798, 2016.

33. Elisaf M, Kitsos G, Bairaktari E, Kalaitzidis R, Kalogeropoulos C and Psilas K: Metabolic abnormalities in patients with primary open-angle glaucoma. Acta Ophthalmol Scand 79: 129-132, 2001.

This work is licensed under a Creative Commons Attribution-NonCommercial-NoDerivatives 4.0 International (CC BY-NC-ND 4.0) License. 